\title{
Laminin $\alpha 5$ modulates fibroblast proliferation in epidural fibrosis through the PI3K/AKT/mTOR signaling pathway
}

\author{
PENGRAN LIU ${ }^{1}$, HUI CHEN ${ }^{2}$, LIANQI YAN ${ }^{3}$ and YU SUN ${ }^{3}$ \\ ${ }^{1}$ Department of Orthopedics, The Second Affiliated Hospital of Xiangya School of Medicine, \\ Central South University, Changsha, Hunan 410012; ${ }^{2}$ Department of Orthopedics, Dalian Medical University, \\ Dalian, Liaoning 116000; ${ }^{3}$ Department of Orthopedics, Clinical Medical College of Yangzhou University, \\ Northern Jiangsu People's Hospital, Yangzhou, Jiangsu 225001, P.R. China
}

Received June 17, 2019; Accepted November 22, 2019

DOI: $10.3892 / \mathrm{mmr} .2020 .10967$

\begin{abstract}
Lumbar laminectomy is commonly deemed as the most valid surgery for a series of lumbar illnesses, such as lumbar disc herniation, which could lead to spinal canal stenosis. However, epidural fibrosis is one of the most common complications that limits the application of lumbar laminectomy, which is mainly caused by proliferation of local fibroblasts. Laminins are glycoproteins that consist of $\alpha, \beta$ and $\gamma$ chains, which serve a crucial role in biological cell behaviors, such as adhesion, differentiation, migration and proliferation, especially the isoform with the fifth $\alpha$ chain-laminin $\alpha 5$. The PI3K/AKT/mTOR signaling pathway was demonstrated to be associated with various biological functions in cells. The aim of the present study was to explore whether laminin $\alpha 5$ is an important factor in epidural fibrosis by modulating the proliferation of fibroblasts through the activation of PI3K/AKT/mTOR signaling pathway. In the animal model, the results of the hematoxylin-eosin staining, cell counting, Masson's trichrome staining and immunohistochemical staining showed laminin $\alpha 5$ to be positively associated with epidural fibrosis. Furthermore, to verify the assumption that laminin $\alpha 5$ could modulate fibroblast proliferation through the PI3K/AKT/mTOR signal pathway, fibroblasts were transfected with laminin $\alpha 5$-small interfering (si)RNA. The results of western blotting (proliferating cell nuclear antigen and cyclin D1), the Cell Counting Kit-8 and EdU incorporation assays indicated that the proliferative level of fibroblasts decreased, and the expression of phosphorylated (p)-focal adhesion kinase 1, p-AKT and p-mTOR was reduced. Subsequently, laminin $\alpha 5$ was overexpressed and the change
\end{abstract}

Correspondence to: Professor Lianqi Yan or Professor Yu Sun, Department of Orthopedics, Clinical Medical College of Yangzhou University, Northern Jiangsu People's Hospital, 98 Nantong West Road, Yangzhou, Jiangsu 225001, P.R. China

E-mail: sbyyylq@csu.edu.cn

E-mail: docsunyu@126.com

Key words: laminin $\alpha 5$, epidural fibrosis, fibroblast, proliferation in cell proliferation and expression of associated proteins contrasted with that observed in siRNA. The results demonstrated that laminin $\alpha 5$ could interfere the activation of the $\mathrm{PI} 3 \mathrm{~K} / \mathrm{AKT} / \mathrm{mTOR}$ signaling pathway. Finally, the inhibition of the PI3K/AKT/mTOR signaling pathway by LY294002 resulted in decreased fibroblast proliferation. In conclusion, laminin $\alpha 5$ could modulate fibroblast proliferation in epidural fibrosis through the PI3K/AKT/mTOR signaling pathway.

\section{Introduction}

In the current medical field, the lumbar laminectomy is deemed the most valid treatment for lumbar illnesses; these include lumbar disc herniation and other associated diseases, which may result in spinal canal stenosis. However, due to inaccurate recognition and inadequate treatment, following surgery the formation of fibrosis on local dura and lumbosacral adhesive arachnoiditis occurs (1). With epidural fibrosis patients often experience chronic lower back and leg pain, as well disability (2). There is also a study that indicated that the function of local inflammatory factors and hematoma organization could be primary contributors of epidural fibrosis (3). Additionally, further studies have illustrated that the excessive proliferation of fibroblasts in the operative region is the most significant element of local fibrosis formation $(4,5)$. Therefore, the methods of inhibiting fibroblast proliferation to reduce fibrosis have become an attractive area of study and previous studies have demonstrated positive results (6-8). However, there are numerous limitations for clinical application, hence further research is still required to solve the problem completely.

Laminins are a type of biofunctional glycoprotein in the extracellular matrix, which consist of three different polypeptide $\alpha, \beta$ and $\gamma$ chains with disulfide bonds; there are five kinds of $\alpha$ chains $(\alpha 1-\alpha 5)$, three $\beta$ chains ( $\beta 1-\beta 3)$ and three $\gamma$ chains $(\gamma 1-\gamma 3)$ (9-11). Furthermore, some studies have shown that all fifteen different laminin trimer-formations are associated with multiple cell biological behaviors, such as adhesion, differentiation, migration and proliferation in various cell lines (12-16). For further research, as the major constituent protein of the extracellular matrix, most laminins extensively express the $\alpha 5$ chain (referred as laminin $\alpha 5$ ), which has a greater influence on the aforementioned cell 
behaviors than the other chains $(17,18)$. Studies have also indicated that laminin $\alpha 5$ is a crucial constituent of the basement membrane in some tissues and organs, such as the skin, hair, lung, intestines and kidney, and plays a significant role in the organism (19). For instance, in mice experiments laminin $\alpha 5$ was found to accelerate the morphogenesis of embryonic skin and hair (20). According to previous studies, laminin $\alpha 5$ was also demonstrated to be associated with human myasthenia, ligament lesions, dermopathy, visual impairment, scar formation malabsorption and vitreous detachment $(21,22)$. Thus, laminin $\alpha 5$ is of great significance, however there are no studies to the best of our knowledge, involving laminin $\alpha 5$, fibroblast and epidural fibrosis until now.

The PI3K/AKT/mTOR signaling pathway is a classical pathway, which has been demonstrated to be associated with various biological cell behaviors including proliferation $(23,24)$. There was also research indicated that AKT pathway is probably involved with epidural fibrosis (25). However, there are few further studies on the signaling pathway involved in fibroblast proliferation and epidural fibrosis. Therefore, the present study explored whether laminin $\alpha 5$ is associated with epidural fibrosis and modulates fibroblast proliferation through the activation of the PI3K/AKT/mTOR signaling pathway. The results of the present study may aid the development of a novel treatment for the prevention of epidural fibrosis.

\section{Materials and methods}

Animals. A total of 40 Sprague-Dawley male rats (mean weight, $250 \mathrm{~g}$; age, 8 weeks) were provided by the Medical College of Yangzhou University (Yangzhou, China). The rats were acclimatized for a week to adapt to the laboratory environment of $23 \pm 2^{\circ} \mathrm{C}$ and $50-60 \%$ humidity, with a 12 -h light/dark cycle and free access to food and water. Rats were then randomly divided into two groups: 2-week group and 4-week group (the number represents the postoperative euthanasia-time; 20 rats per group). During the preparation process, all rats were appropriately treated according to the standards of International Laboratory Animal Care.

Animal laminectomy model. To simulate the situation of clinical patients, lumbar laminectomy was carefully performed in all rats according to a laminectomy model and the procedure was conducted as previously reported (26). Following the application of $1 \%$ pentobarbital sodium for anesthesia $(40 \mathrm{mg} / \mathrm{kg})$, the rats were shaved on the back among the first and second lumbars (L1 and L2) to expose the operative area clearly and the local skin was sterilized three times with iodine. The local connective tissue and muscles were separated layer by layer until the vertebral plate, and the plates of L1 and L2 were carefully removed with a rongeur to expose the spinal cord distinctly, avoiding causing any other injuries. Following thorough hemostasis, the wound was sutured in mattress-style with Coated Vicryl Plus Antibacterial Suture (Johnson Co., Ltd). All procedures aforementioned were performed in sterile conditions by professional veterinarians with the certification from Jiangsu Experimental Animal Association (Jiangsu, China).

Histological analysis of hematoxylin and eosin $(H \& E)$ and Masson trichrome stains. After lumbar laminectomy operation, the two groups of rats were individually euthanized at 2 and 4 weeks to perform histological analysis to detect the degree of local fibrosis. The rats were anesthetized with $1 \%$ pentobarbital sodium and perfused with $4 \%$ paraformaldehyde intracardially for euthanasia. Subsequently, the L1 and L2 lumbar column with local muscles and epidural fibrosis were excised and fixed with $10 \%$ buffered formalin for one week at room temperature, then immersed in Ethylene Diamine Tetraacetic Acid (EDTA) for 40 days for decalcification. Finally, the columns were embedded in paraffin and then sliced into successive 4- $\mu \mathrm{m}$ transverse sections.

For H\&E staining, the sections were subsequently stained with hematoxylin for $5 \mathrm{~min}$ and then eosin for $5 \mathrm{~min}$, both at room temperature. For Masson trichrome staining, the sections were subsequently immersed in $50 \%$ potassium dichromate overnight at room temperature, stained with hematoxylin for $3 \mathrm{~min}$ at room temperature and incubated in Ponceau $\mathrm{S}$ dye for $5 \mathrm{~min}$ at room temperature. Then, the sections were washed and incubated with $1 \%$ phosphomolybdic acid for $2 \mathrm{~min}$ at room temperature prior to being stained with aniline blue for $5 \mathrm{~min}$ at room temperature. The degree of fibrosis, local fibroblast counting, and the content of epidural collagen were observed by optical photographic light microscopy at x40 and x200 magnification. Stained cells were counted in three random views of fibrotic area per section by Image Pro Plus 6.0 software (Media Cybernetics, Inc.).

Immunohistochemistry. Following the initial fixing steps described above and obtaining paraffin-embedded sections (4 $\mu \mathrm{m})$, immunohistochemistry analysis of laminin $\alpha 5$ protein expression was performed with a Ready-to-use HP IHC detection kit (Absin Bioscience, Inc.), according to the manufacturer's protocol. Briefly, sections of each group underwent antigen retrieval in sodium citrate at $100^{\circ} \mathrm{C}$ for $20 \mathrm{~min}$. Sections were subsequently deparaffinized in xylene at room temperature and rehydrated in a descending alcohol series (100, 85 and 75\%), and blocked in 100\% FBS (Gibco; Thermo Fisher Scientific, Inc.) for $15 \mathrm{~min}$ at room temperature. Sections were subsequently incubated with the laminin $\alpha 5$ primary antibody (1:200; cat. no. NBP2-42391; Novus Biologicals, Ltd.) at $4^{\circ} \mathrm{C}$ overnight, then incubated with the secondary antibody included in the kit at room temperature for $2 \mathrm{~h}$. Finally, the sections were stained with DAB reagent for $2 \mathrm{~min}$ at room temperature and then, hematoxylin for $2 \mathrm{~min}$ at room temperature. Stained cells were observed under an optical photographic light microscope at x200 magnification and analyzed by Image Pro Plus 6.0 software (Media Cybernetics, Inc.).

Culture and treatment of fibroblasts. The human fibroblasts were obtained from Shanghai Cell Repository of the Chinese Academy of Sciences. Cells were cultured at $37^{\circ} \mathrm{C}$ in $5 \% \mathrm{CO}_{2}$ with DMEM (Gibco; Thermo Fisher Scientific, Inc.), supplemented with $15 \%$ FBS (Gibco; Thermo Fisher Scientific, Inc.) and $1 \%$ penicillin \& streptomycin (Gibco; Thermo Fisher Scientific, Inc.). A total of $1 \times 10^{6}$ fibroblasts were seeded in petri dishes with a variety of specifications overnight until a confluence of $70 \%$ was attained; then the dishes were washed twice with PBS. One third of the fibroblasts were set as the LY group and were treated with the PI3K inhibitor LY294002 
(MedChemExpress) diluted in dimethylsulfoxide to $50 \mu \mathrm{M}$ for $24 \mathrm{~h}$. Another third of the fibroblasts were treated with siRNA for knockdown or lentiviral vectors for overexpression. All fibroblasts were maintained in the growth phase between 3 and 6 passages.

Small interfering (si)RNA. siRNA of laminin $\alpha 5$ (5'-GCA TCAGCTTCGACAGTCA-3') and the negative control (cat. no. siN0000001-1-5; with same sequence length as siRNA-laminin $\alpha 5$ but non-targeting) were purchased from Guangzhou RiboBio, Co., Ltd. The fibroblasts were transfected with $50 \mathrm{nM}$ siRNA at a confluence of $70 \%$ for $48 \mathrm{~h}$ with Opti-MEM (Gibco; Thermo Fisher Scientific, Inc.) and Lipofectamine ${ }^{\circledR} 2000$ reagent (Invitrogen; Thermo Fisher Scientific, Inc.), following the manufacturer's protocol. The efficiency of transfection was detected by reverse transcription-quantitative PCR (RT-qPCR) and immunofluorescence. Transfected cells were maintained in culture for $48 \mathrm{~h}$ prior to subsequent experiments.

Gene overexpression by lentiviral infection. The GV418 and GV419 lentiviral vectors for overexpressing the laminin $\alpha 5$ target gene and the scramble control (empty vector) were obtained from Shanghai Genechem Co., Ltd. Lentiviral infection was performed to overexpress laminin $\alpha 5$ following the manufacturer's protocol. Fibroblasts in the overexpression and scramble control group were cultured until they reached $70 \%$ confluence. Subsequently, $1 \times 10^{6}$ fibroblasts/well were transfected with $2 \times 10^{7} \mathrm{TU}$ (multiplicity of infection of 20) laminin $\alpha 5$ GV418 lentiviral vector or GV418 scramble control empty vector overnight in the presence of $2 \mathrm{mg} / \mathrm{ml}$ polybrene (Gibco; Thermo Fisher Scientific, Inc.) and then replaced with fresh complete medium. After $48 \mathrm{~h}$-transfection at $37^{\circ} \mathrm{C}$, cells were cultured in puromycin (Sigma-Aldrich; Merck KGaA) at a concentration of $2 \mu \mathrm{g} / \mathrm{ml}$ for $72 \mathrm{~h}$ for the preliminary screening to eliminate non-transfected cells.

Subsequently, the laminin $\alpha 5$ overexpression GV419 lentiviral and the GV419 scramble control empty vector were transfected into $1 \times 10^{6}$ fibroblasts as described previously. Then, fibroblasts were screened with $400 \mu \mathrm{g} / \mathrm{ml}$ G418 Sulfate (Thermo Fisher Scientific, Inc.) for $72 \mathrm{~h}$. The transfection efficiency was verified as aforementioned, with an untreated group used as the control group, and cells were kept in culture for subsequent experiments.

$R N A$ preparation and $R T-q P C R$. Total RNA was extracted from fibroblasts with TRIzol ${ }^{\circledR}$ reagent (Thermo Fisher Scientific, Inc.), according to the manufacturer's protocol. Reverse transcription to cDNA was performed using the FastKing DNA Dispelling RT SuperMix (Tiangen Biotech Co., Ltd.), according to the manufacturer's protocol. The RT conditions were $42^{\circ} \mathrm{C}$ for $15 \mathrm{~min}$ and $95^{\circ} \mathrm{C}$ for $3 \mathrm{~min}$. All qPCR reactions were run on the StepOnePlus Real-Time PCR System (Thermo Fisher Scientific, Inc.) with a SYBR ${ }^{\circledR}$ Green Master Mix kit (Vazyme Biotech Co. Ltd), according to the manufacturer's protocol. The primers used are displaying in Table I. The following thermocycling conditions were used for the qPCR: Initial denaturation at $95^{\circ} \mathrm{C}$ for $5 \mathrm{~min}$; 40 cycles at $95^{\circ} \mathrm{C}$ for $10 \mathrm{sec}$ and $60^{\circ} \mathrm{C}$ for $30 \mathrm{sec}$; and $95^{\circ} \mathrm{C}$ for $15 \mathrm{sec}$, $60^{\circ} \mathrm{C}$ for $60 \mathrm{sec}$ and $95^{\circ} \mathrm{C}$ for $15 \mathrm{sec}$. Expression levels were
Table I. Primers for reverse transcription-quantitative PCR.

\begin{tabular}{ll} 
Gene & \multicolumn{1}{c}{ Primer sequence $\left(5^{\prime} \rightarrow 3^{\prime}\right)$} \\
\hline Laminin $\alpha 5$ & F: TGCACCCGCCCTACTTCAA \\
& R: GGGTGACGTTGACCTCGTTGTA \\
GAPDH & F: GAAGCTTGTCATCAATGGAAAT \\
& R: TGATGACCCTTTTGGCTCCC
\end{tabular}

F, forward; R, reverse.

quantified using the $2^{-\Delta \Delta \mathrm{Cq}}$ method (27) and normalized to the loading control GAPDH.

Western blot analysis. A total of $3 \times 10^{6}$ fibroblasts were lysed on ice with RIPA lysis buffer (Beyotime Institute of Biotechnology), according to the manufacturer's protocol. Total protein concentration was determined with a bicinchoninic acid protein assay kit (Thermo Fisher Scientific, Inc.). Western blot analysis was performed as previously reported (28). Briefly, $30 \mu \mathrm{g}$ protein/lane was separated via $10 \%$ SDS-PAGE and transferred onto a PVDF membrane. The membranes were blocked with 5\% skim milk in TBS and $0.05 \%$ Tween-20 for $2 \mathrm{~h}$ at room temperature and subsequently incubated with primary antibodies at $4{ }^{\circ} \mathrm{C}$ overnight. The primary antibodies were against proliferating cell nuclear antigen (PCNA; 1:1,000; cat. no. 13110; Cell Signaling Technology, Inc.), cyclin D1 (1:1,000; cat. no. 55506; Cell Signaling Technology, Inc.), phosphorylated (p)-focal adhesion kinase 1 (FAK1; 1:1,000; cat. no. 3281; Cell Signaling Technology, Inc.), FAKT1 (1:1,000; cat. no. 71433; Cell Signaling Technology, Inc.), AKT (1:1,000; cat. no. 4685; Cell Signaling Technology, Inc.), p-AKT (1:1,000; cat. no. 4060; Cell Signaling Technology, Inc.), mTOR (1:1,000; cat. no. 2983; Cell Signaling Technology, Inc.), p-mTOR (1:1,000; cat. no. 5536; Cell Signaling Technology, Inc.) and GAPDH (1:1,000; cat. no. 5174; Cell Signaling Technology, Inc.). Subsequently, the membranes were incubated with a horseradish peroxidase-conjugated anti-rabbit IgG secondary antibody (1:2,000; cat. no. 7074; Cell Signaling Technology, Inc.) for $2 \mathrm{~h}$ at room temperature. The target protein expression was detected with ECL reagents (Beyotime Institute of Biotechnology) using a ChemiDoc XRSp system (Bio-Rad Laboratories, Inc.). The results were analyzed using ImageJ version 1.46r software (National Institutes of Health).

Immunofluorescence staining. A total of $2.5 \times 10^{5}$ fibroblasts from each group were simultaneously cultured in 24-well plates overnight until $70 \%$ confluent. Then cells were fixed with $4 \%$ polyoxymethylene in PBS at room temperature for $15 \mathrm{~min}$, then immersed in $0.1 \%$ Triton X-100. Subsequently, the sections were blocked in 3\% BSA (Gibco; Thermo Fisher Scientific, Inc.) for $30 \mathrm{~min}$ at room temperature and incubated with anti-laminin $\alpha 5$ primary antibody (1:100; cat. no. 220399; Abcam) overnight at $4^{\circ} \mathrm{C}$ and probed with a FITC-conjugated goat anti-rabbit $\mathrm{IgG}$ secondary antibody (1:200; cat. no. 33112ES60; Yeasen Biotechnology (Shanghai) Co., Ltd.) for $2 \mathrm{~h}$ at room temperature. Finally, the cell nuclei were stained with Hoechst for $5 \mathrm{~min}$ at room temperature, 

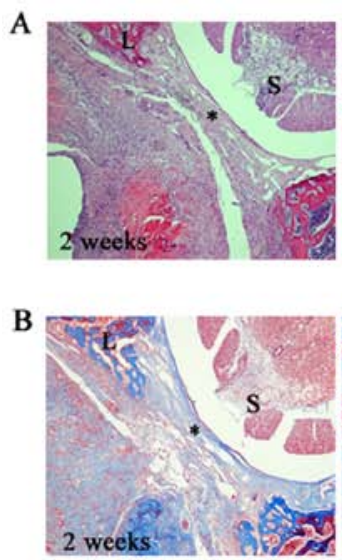
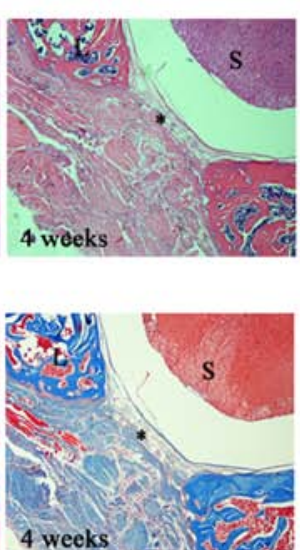
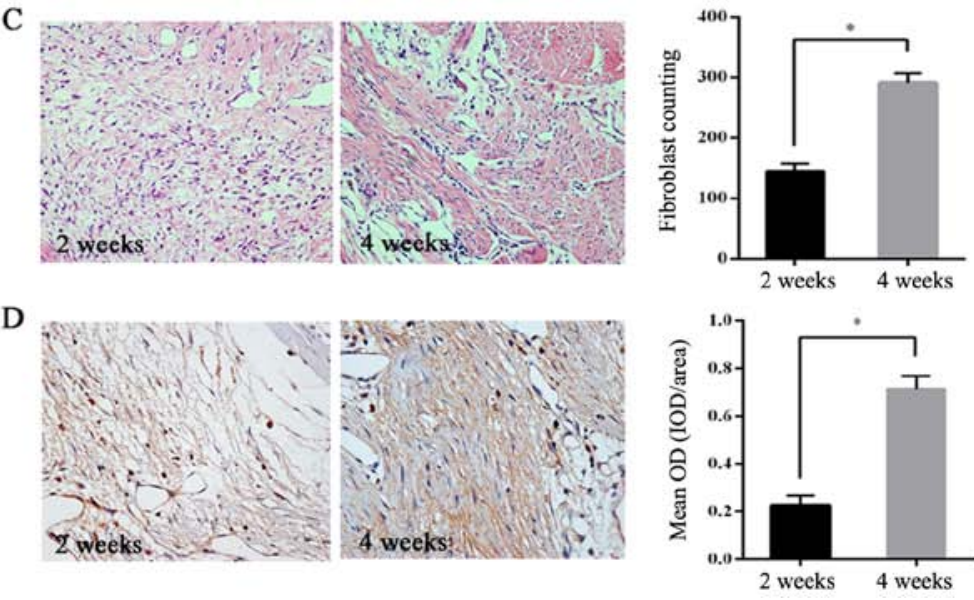

Figure 1. Laminin $\alpha 5$ expression is related to epidural fibrosis. (A) In H\&E staining, excessive fibrosis '*' with thick adherence to spinal dura was observed in the 4 weeks group. In the 2 weeks group, there were notably fewer areas of fibrosis, which was observed in a time-dependent manner. In the images, the surgical area is marked by ' $\mathrm{L}$ ' and the spinal cord by 'S'. Magnification, $\mathrm{x} 40$. (B) In Masson's trichrome staining, collagen is indicated in royal blue. Images indicated that epidural collagen was gradually synthesized with increasing time. Magnification, $\mathrm{x} 40$. (C) H\&E staining images showed that the number of fibroblasts within the surgical area around the spinal dura increased with time, as shown in the histogram. Magnification, $x 200$. The data are presented as the mean \pm SD of the two groups. ${ }^{*} \mathrm{P}<0.05$. (D) Immunohistochemical staining of laminin $\alpha 5$ in epidural fibrosis tissues. The results of laminin $\alpha 5$ expression are shown as the mean integral OD in the histogram. Magnification, x200. Analysis was conducted using Image Pro Plus 6.0 (Media Cybernetics, Inc.). The data are presented as the mean $\pm \mathrm{SD}$ of two groups, ${ }^{*} \mathrm{P}<0.05$. SD, standard deviation; H\&E, hematoxylin and eosin; OD, optical density.
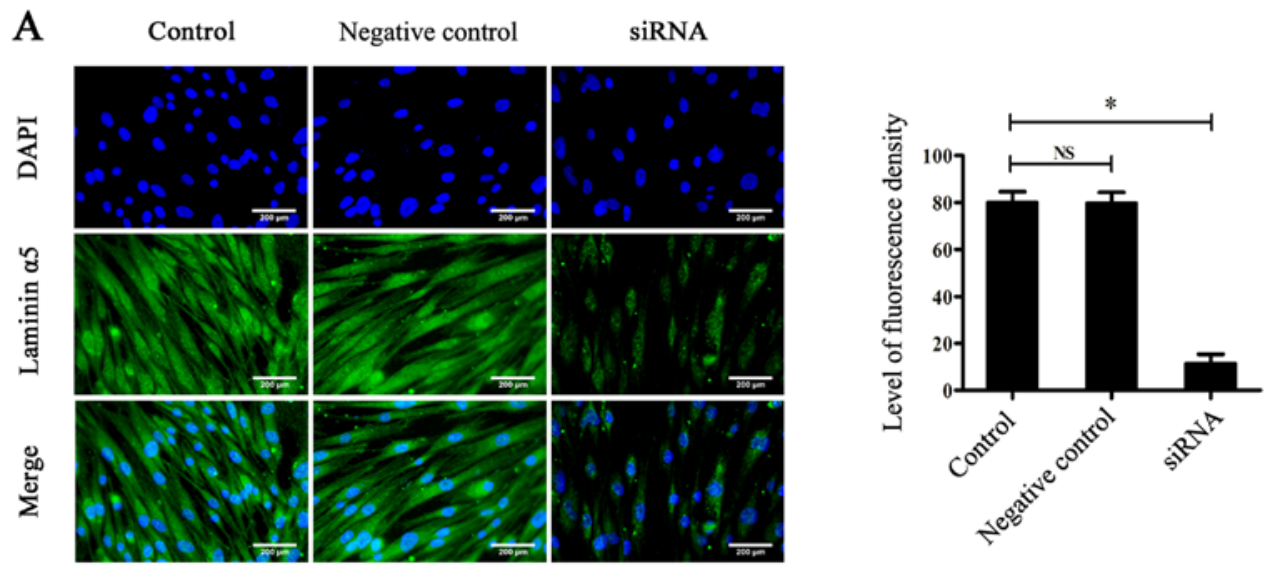

B

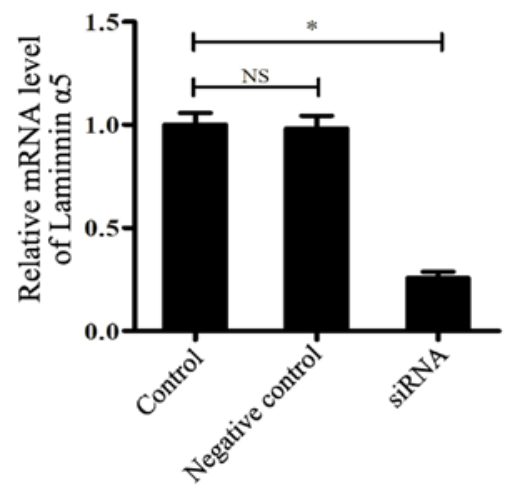

Figure 2. Efficiency of laminin $\alpha 5$ siRNA-knockdown by RT-qPCR and immunofluorescence. (A) The immunofluorescence assay of laminin $\alpha 5$ indicated that the fluorescence density level of the siRNA group was significantly decreased compared with the control group. No statistical significance was observed among the control and negative control groups. The data are presented as the mean \pm SD of three independent groups. ${ }^{*} \mathrm{P}<0.05$. (B) RT-qPCR indicated a downregulated mRNA level in the siRNA group. GAPDH was used as the control. The data were presented as the mean \pm SD of three independent groups. ${ }^{*} \mathrm{P}<0.05$. SD, standard deviation; RT-q, reverse transcription-quantitative; si, small interfering; NS, not significant; DAPI, 4',6-diamidino-2-phenylindole.

then observed with a Zeiss inverted fluorescence microscope (magnification, $\mathrm{x} 200$ ) to determine the expression levels of the target protein. The data were analyzed using Image Pro Plus 6.0 (Media Cybernetics, Inc.). 
A
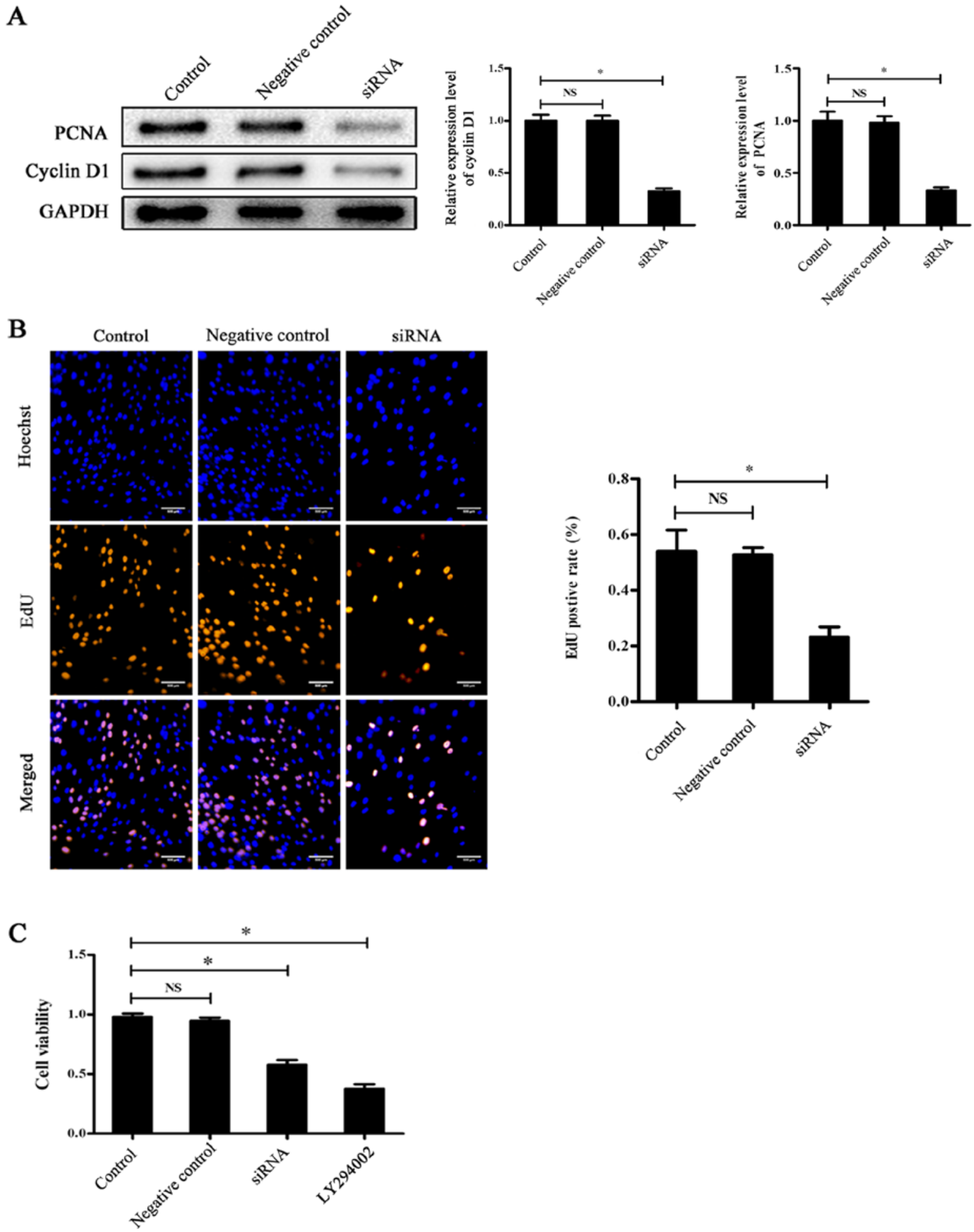

Figure 3. Knockdown of laminin $\alpha 5$ decreases fibroblast proliferation. (A) Western blotting assays demonstrated that the expression of PCNA and cyclin D1 in the siRNA group were increased compared with in the control group. GAPDH was set as the control. The data are presented as the mean \pm SD of three independent groups. ${ }^{*} \mathrm{P}<0.05$. (B) Fibroblast proliferation level was detected by EdU incorporation assay. Positive rate was shown as the mean \pm SD. ${ }^{*} \mathrm{P}<0.05$. (C) Viability was determined by Cell Counting Kit- 8 assay. ${ }^{*} \mathrm{P}<0.05$. si, small interfering RNA; PCNA, proliferating cell nuclear antigen; NS, not significant.

Cell viability. Cell viability was analyzed using the Cell Counting Kit-8 assay (CCK-8; cat. no. CK04; Dojindo Molecular Technologies, Inc.), according to the manufacturer's protocol. Fibroblasts were cultured in triplicate in 96-well plates for $24 \mathrm{~h}$ at $37^{\circ} \mathrm{C}$, then treated with $10 \mu \mathrm{l} \mathrm{CCK}-8$ reagent for $2 \mathrm{~h}$ at $37^{\circ} \mathrm{C}$. The optical density value at $450 \mathrm{~nm}$ was determined with a microplate absorbance reader (Bio-Tek;
Elx800). The cell survival rate was calculated according to the manufacturer's specification.

EdU incorporation assay. The EdU incorporation assay was conducted to evaluate fibroblast proliferation. The kFlour555 Click-iT EdU kit was obtained from KeyGen Biotech Co., Ltd. A total of $2.5 \times 10^{5}$ fibroblasts were cultured in 24-well plates 

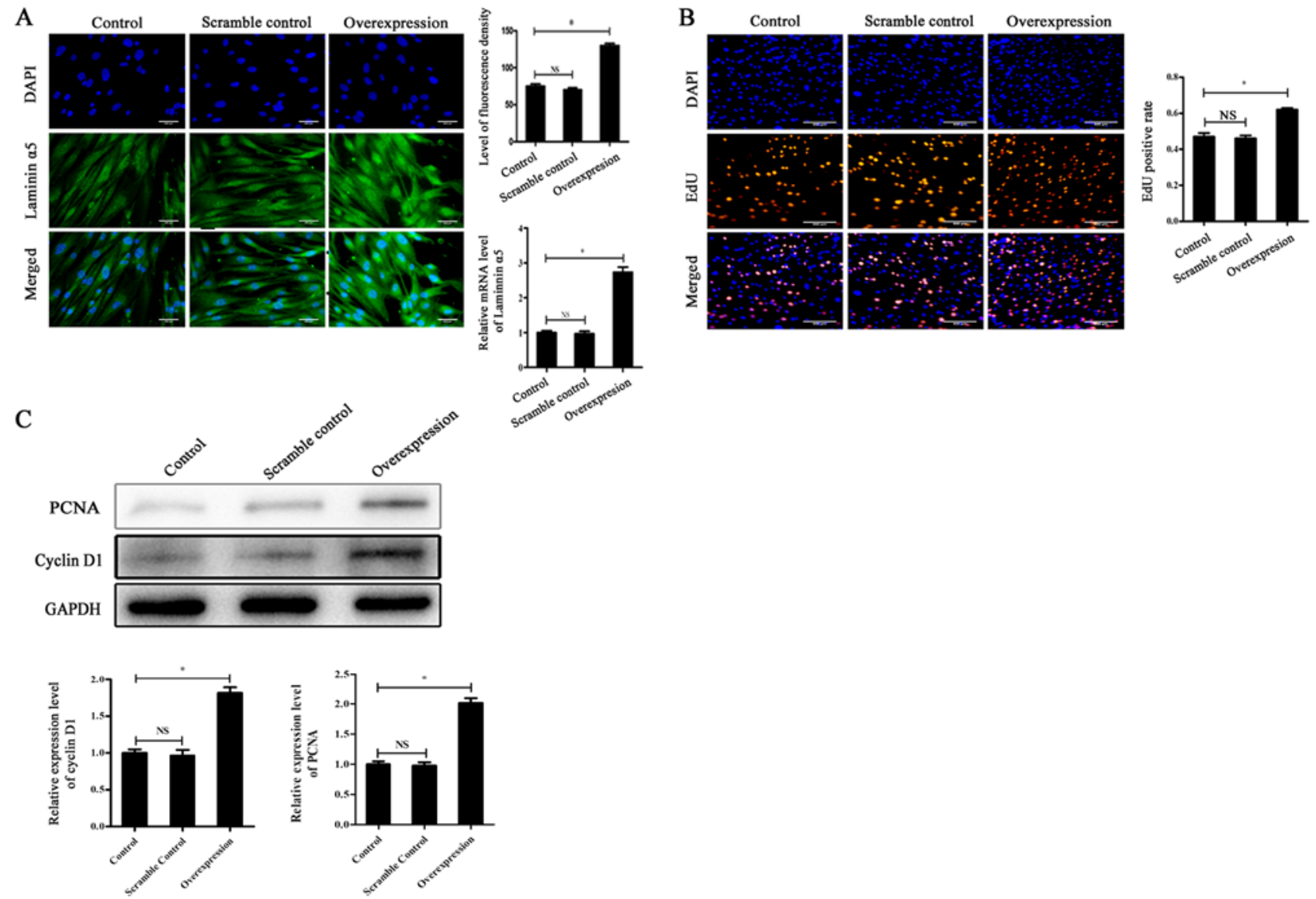

Figure 4. Overexpression of laminin $\alpha 5$ promotes fibroblast proliferation. (A) Efficiency of laminin $\alpha 5$ overexpression by reverse transcription-quantitative PCR and immunofluorescence assays. The data are presented as the mean \pm SD of three independent groups. ${ }^{*} \mathrm{P}<0.05$. (B) Fibroblast proliferation level was detected by EdU incorporation assay. The positive rate is shown as the mean $\pm \mathrm{SD}$. " $\mathrm{P}<0.05$. (C) Western blot assay of PCNA and cyclin D1 levels. GAPDH was set as the control. The data are presented as the mean \pm SD of three independent groups. ${ }^{*} \mathrm{P}<0.05$. SD, standard deviation; PCNA, proliferating cell nuclear antigen; NS, not significant; DAPI, 4',6-diamidino-2-phenylindole.

for $24 \mathrm{~h}$ until $70 \%$ confluent. Then cells were subsequently incubated in $10 \mu \mathrm{mol} / 1 \mathrm{EdU}$ working solution for $2 \mathrm{~h}$ at $37^{\circ} \mathrm{C}$, fixed in $4 \%$ polyoxymethylene for $30 \mathrm{~min}$ at room temperature and incubated with $0.5 \%$ Triton $\mathrm{X}-100$ for $20 \mathrm{~min}$ in the dark at room temperature. After immerged in Click-iT mixture system, cell nuclei were stained with Hoechst 33342 for 5 min at room temperature. Finally, the cells were observed under a Zeiss inverted fluorescence microscope (magnification, x200). Orange was deemed as a positive signal of proliferation and the cell nucleus was royal blue. The positive EdU rate was calculated using ImageJ software.

Statistical analysis. The data of the present study are presented as the mean \pm SD and statistical analysis was performed using SPSS 19.0 statistical software (IBM Corp.). Each experiment was performed in triplicate. The significance of the differences among groups was evaluated by Student's t-test or one-way ANOVA followed by Tukey's post hoc test. $\mathrm{P}<0.05$ was considered to indicate a statistically significant difference.

\section{Results}

Laminin $\alpha 5$ is positively associated with epidural fibrosis. To detect epidural fibroblast density and fibrosis, histological analysis by $H \& E$ staining, fibroblast counting, and Masson trichrome stains were performed. As shown in Fig. 1A and C, from $H \& E$ staining and fibroblast counting, extensive fibrosis and significantly high densities of fibroblasts were found in the postoperative area in the 4-week group compared with the 2-week group $(\mathrm{P}<0.05)$. Similarly, the Masson trichrome stains (Fig. 1B) indicated that the presence of collagen in local tissue on the dura mater of the 4-week group was markedly increased compared with the 2-week group. The results also demonstrated that the epidural fibrosis level and fibroblast density in the laminectomy area were increased in a time-dependent manner. This supports the previous conclusion that the increased presence of fibroblasts in the operative region is a significant cause of epidural fibrosis. To determine whether laminin $\alpha 5$ is involved in epidural fibrosis, its expression in the two groups was further assessed, as shown in Fig. 1D. The laminin $\alpha 5$ content was significantly increased in the 4-week group compared with the 2 -week group $(\mathrm{P}<0.05)$. These results supported the assumption that laminin $\alpha 5$ may be positively associated with epidural fibrosis.

Laminin $\alpha 5$ modulates fibroblast proliferation. Based on the results of the animal model, the effect of laminin $\alpha 5$ on fibroblast proliferation was further studied. The fibroblasts were transfected with laminin $\alpha 5$ siRNA, which was followed with RT-qPCR and immunofluorescence to detect the transfection 

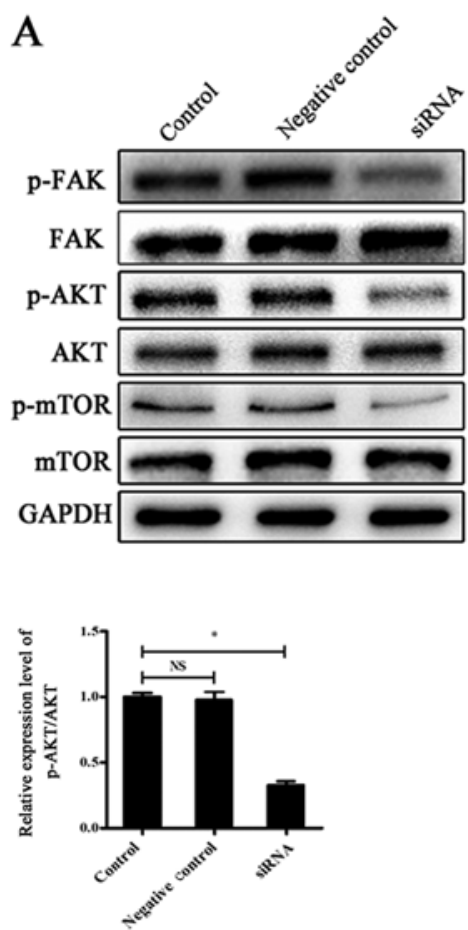
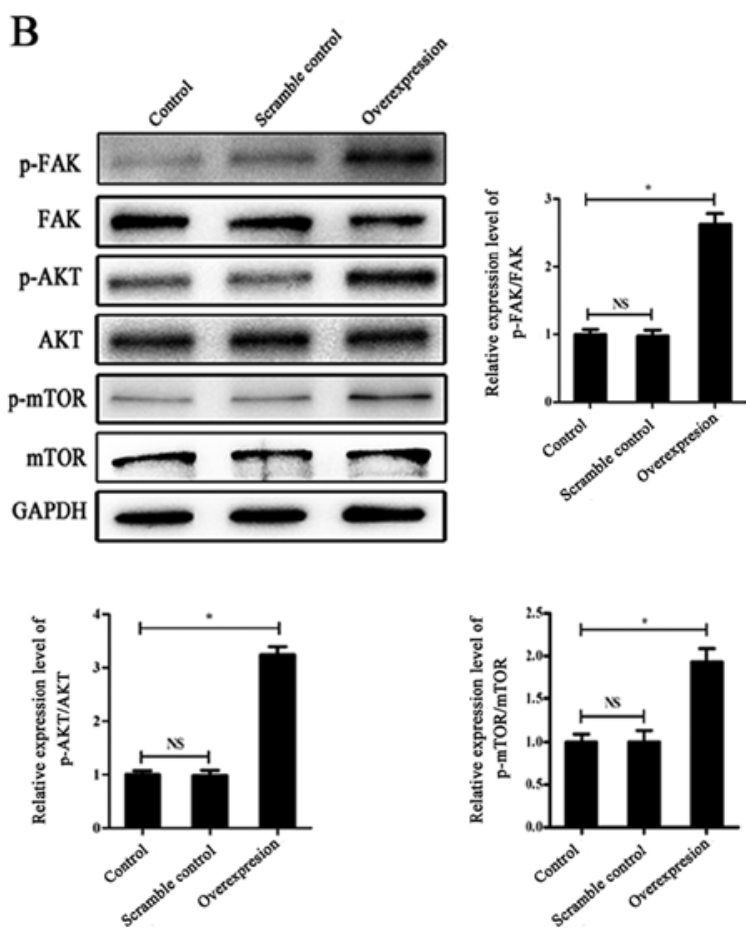

Figure 5. Laminin $\alpha 5$ interferes with the activation of the PI3K/AKT/mTOR signaling pathway. (A) Knockdown of laminin $\alpha 5$ reduces the activation of the PI3K/AKT/mTOR signaling pathway. Western blotting of p-FAK, FAK, p-AKT, AKT, p-mTOR and mTOR expression levels in fibroblasts within the control, siRNA and negative control groups. GAPDH was set as the control. The data are presented as the mean \pm SD of three independent experiments. ${ }^{*} \mathrm{P}<0.05$. (B) Overexpression of laminin $\alpha 5$ promotes the activation of the PI3K/AKT/mTOR signaling pathway. Western blot assay of p-FKA, FAK, p-AKT, AKT, p-mTOR and mTOR expression levels in fibroblasts within the control, scramble control and overexpression group. GAPDH was set as the control. The data are presented as the mean $\pm \mathrm{SD}$ of three independent groups. "P<0.05. SD, standard deviation; si, small interfering RNA; p, phosphorylated; FAK, focal adhesion kinase; AKT, protein kinase B; mTOR, mammalian target of rapamycin; PI3K, phosphoinositide 3 kinase; NS, not significant.

efficiency. As shown in Fig. 2A, immunofluorescence staining indicated that the expression of laminin $\alpha 5$ in the siRNA group was significantly reduced after siRNA-knockdown compared with the control group $(\mathrm{P}<0.05)$, which was further demonstrated by RT-qPCR (Fig. 2B). After transfection, the PCNA and cyclin D1 (marker of cell proliferation) levels were determined by western blotting. The results demonstrated that the expression levels of PCNA and cyclin D1 were significantly reduced in the siRNA group compared with the control group ( $\mathrm{P}<0.05$; Fig. $3 \mathrm{~A})$. An EdU incorporation assay was subsequently performed to further study the proliferative level, which demonstrated that the positive rate of proliferation was also significantly decreased in the siRNA group compared with the control group $(\mathrm{P}<0.05$; Fig. $3 \mathrm{~B})$. The results of the CCK-8 assay also demonstrated that the cell viability of the siRNA group was significantly reduced compared with the control group ( $\mathrm{P}<0.05$; Fig. $3 \mathrm{C}$ ). Subsequently, laminin $\alpha 5$ was overexpressed by lentiviral vectors to perform the same experiments. The transfection efficiency was confirmed by RT-qPCR and immunofluorescence (Fig. 4A). The EdU positive rate and the expression levels of PCNA and cyclin D1 were significantly higher in the overexpression group compared to the control group (Fig. 4B and C). This indicated that the proliferative rate of cells following laminin $\alpha 5$ overexpression was increased compared with the control group, which was opposite to the results obtained with siRNA (Fig. 4B and C). Collectively, these results suggested that laminin $\alpha 5$ may be associated with fibroblast viability and it could modulate cell proliferation.
Laminina5interfereswith theactivationofthe PI3K/AKT/mTOR signaling pathway. After transfection with laminin $\alpha 5$ siRNA, western blotting was performed to determine the influence of target proteins of the PI3K/AKT/mTOR signaling pathway. The results indicated that after gene knockdown, the expression ratio of $\mathrm{p}-\mathrm{AKT} / \mathrm{AKT}$ and $\mathrm{p}-\mathrm{mTOR} / \mathrm{mTOR}$ significantly decreased compared with the control group ( $\mathrm{P}<0.05$; Fig. 5A). Thus, downregulation of laminin $\alpha 5$ prevented the activation of PI3K/AKT/mTOR signaling. The expression level of p-FAK, an upstream protein of the PI3K/AKT/mTOR signaling pathway, was also detected and there was also a significant decrease in the siRNA group compared with the control group $(\mathrm{P}<0.05$; Fig. 5A). Subsequently, the expression levels of proteins in the PI3K/AKT/mTOR signaling pathway in laminin $\alpha 5$-overexpressing fibroblasts were also investigated and the results in Fig. 5B demonstrated a reversed tendency of signaling: The expression ratio of $\mathrm{p}-\mathrm{AKT} / \mathrm{AKT}$ and $\mathrm{p}$-mTOR/mTOR in the overexpression group was significantly increased compared with the control group, as well as the expression ratio of $\mathrm{p}-\mathrm{FAK} / \mathrm{FAK}(\mathrm{P}<0.05)$. All of the results indicated that laminin $\alpha 5$ may interfere with PI3K/AKT/mTOR signaling activation and the expression of $\mathrm{p}-\mathrm{FAK}$.

The PI3K/AKT/mTOR signal pathway regulates fibroblast proliferation. To confirm whether PI $3 \mathrm{~K} / \mathrm{AKT} / \mathrm{mTOR}$ signaling could regulate fibroblast proliferation, cells were treated with the signaling pathway inhibitor LY294002. Following treatment with LY294002 for $24 \mathrm{~h}$, western blotting was performed to detect the expression of PCNA, cyclin D1, 

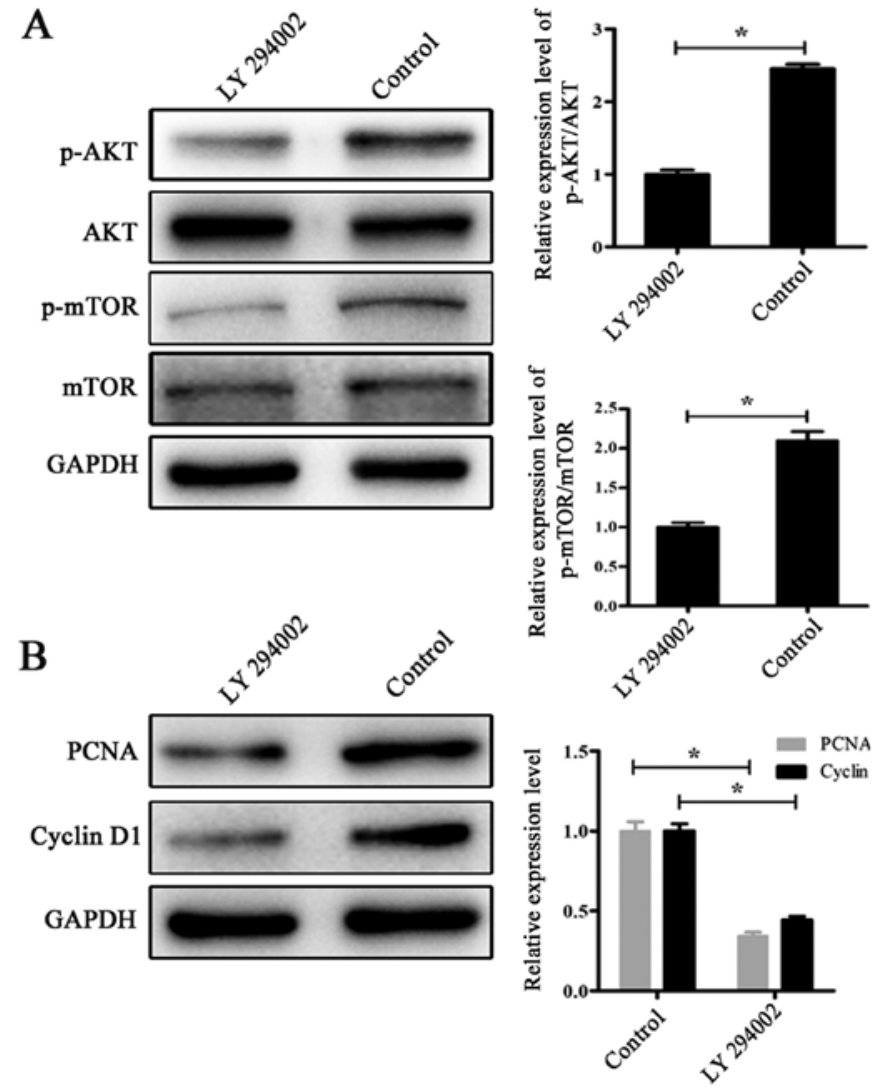

Figure 6. PI3K/AKT/mTOR signaling regulates fibroblast proliferation. (A) Western blot analysis of p-AKT, AKT, p-mTOR and mTOR expression levels in fibroblasts of the control and LY294002 group. GAPDH was set as the control. " $\mathrm{P}<0.05$. (B) Western blotting of PCNA and cyclin D1 expression levels in fibroblasts of control and LY294002 group. GAPDH was set as the control. ${ }^{*} \mathrm{P}<0.05$. SD, standard deviation; p, phosphorylated; PCNA, proliferating cell nuclear antigen.

p-AKT and p-mTOR. As shown in Fig. 6A, the expression ratio of $\mathrm{p}-\mathrm{AKT} / \mathrm{AKT}$ and $\mathrm{p}-\mathrm{mTOR} / \mathrm{mTOR}$ were significantly decreased in the LY294002 group compared with the control group $(\mathrm{P}<0.05)$, which indicated successful inhibition. In Fig. 6B, the downregulation of PCNA and cyclin D1 in the LY294002 group suggested that the PI3K/AKT/mTOR signaling pathway could regulate fibroblast proliferation, which was further proved in the CCK-8 assay (Fig. 3C).

\section{Discussion}

Previous studies have indicated the epidural fibrosis on dura mater after laminectomy operation, which ultimately results in a negative outcome for patients $(8,29)$. Various studies have attempted to solve this problem by local drug applications $(30,31)$ and biomaterials $(32)$. However, there are numerous disadvantages that limit clinical popularization. Therefore, the prevention of epidural fibrosis through the reduction of fibroblast proliferation has been a continuously popular research topic.

The extracellular matrix is a structure with an important role to support cell construction and promote various functions, such as adhesion, differentiation, migration and proliferation, which are also associated with the development of numerous diseases $(33,34)$. The laminins are an important part of the extracellular matrix and serve a primary role in multiple biological behaviors (12). Additionally, there are several studies indicate that the $\alpha 5$ chain (laminin $\alpha 5$ ) is widely expressed in the laminin glycoprotein family, which suggests that laminins may serve important roles in most cell functions $(17,18)$. Further studies also illustrated the involvement of laminin $\alpha 5$ in maintaining the stability of the basement membrane and organ formation, including the placenta during the embryonic phase and dental epithelium growth $(35,36)$. Therefore, laminin $\alpha 5$ is a crucial factor in an organism, so the present study assumed it might also be involved in the formation of epidural fibrosis.

Several studies have illustrated that the PI3K/AKT/mTOR signaling pathway modulates cellular proliferation and various biological behaviors $(23,24)$. PI3K is a bridge factor between extracellular signaling and cellular response effects, where activated PI3K could promote the transformation of AKT, which accelerates the phosphorylation of downstream factor mTOR to inhibit cell apoptosis $(37,38)$. Thus, it is reasonable to assume that laminin $\alpha 5$ could be a pivotal point in epidural fibrosis and modulate fibroblast proliferation through the activation of the PI3K/AKT/mTOR signalling pathway.

In the initiation of the present study, the association between laminin $\alpha 5$ and epidural fibrosis was investigated. There are a series of methods to detect the epidural fibrosis formation, such as H\&E staining, Masson trichrome stains, local fibroblast number counting (7), MR imaging assessment (39) and high-resolution CT scan (1). In the present study histological evaluation was used to assess the epidural fibrosis level. The result from H\&E staining, fibroblast counting and Masson trichrome stains indicated that epidural fibrosis got thicker and local fibroblast number increased over time. On that basis, further immunohistochemistry of laminin $\alpha 5$ showed that the expression was similar to epidural fibrosis formation and presented in a time-dependent manner. It demonstrated that laminin $\alpha 5$ was strongly associated with epidural fibrosis and local fibroblast proliferation.

After animal model experiments, analysis was conducted at the cellular level to study the detail of the mechanism involved in laminin $\alpha 5$ and fibroblast proliferation. Laminin $\alpha 5$ was knocked down for further studies including western blotting, EdU incorporation assay and CCK-8 assay, which showed that fibroblasts following knockdown presented a lower level of proliferation and cell vitality. For further confirmation, the laminin $\alpha 5$ was overexpressed, in which the present study demonstrated an increase in cell proliferation. The results indicated that laminin $\alpha 5$ could modulate fibroblast proliferation, which is similar to previous studies where laminin $\alpha 5$ played a marked role in cell behaviors, such as proliferation $(17,18)$. Further detection of p-AKT/AKT and $\mathrm{p}$-mTOR/mTOR in laminin $\alpha 5$-knockdown fibroblasts implied that the PI3K/AKT/mTOR signal pathway activation was reduced, which dramatically increased following overexpression. Thus, laminin $\alpha 5$ could modulate the activation of PI3K/AKT/mTOR signaling, which corroborates with the finding of another study, that laminin $\alpha 5$ serves a biological role through this signaling pathway (40). Furthermore, the expression of p-FAK decreased after laminin $\alpha 5$ knockdown but increased following its overexpression. FAK is the hub of multiple signal transduction pathways; it can be phosphorylated to an active form by the activation of integrin, which initiates 
multiple signaling pathways including PI3K/AKT/mTOR (41). The change in p-FAK expression in the present study indicated that the mechanism of laminin $\alpha 5$ modulates the activation of the $\mathrm{PI} 3 \mathrm{~K} / \mathrm{AKT} / \mathrm{mTOR}$ signaling pathway might be through integrin and FAK, which was similar to the study of Santos et al (42), although this requires further confirmation. Then after the inhibition of the signaling pathway with LY294002, cell proliferation was decreased, which revealed that the PI3K/AKT/mTOR signaling pathway could regulate fibroblast proliferation. Combined with the results that laminin $\alpha 5$ modulates fibroblast proliferation and interferes the activation of the PI3K/AKT/mTOR signaling pathway, it can be concluded that laminin $\alpha 5$ might modulate fibroblast proliferation in epidural fibrosis through the PI3K/AKT/mTOR signaling pathway.

Fukumoto et al (36) found laminin $\alpha 5$ is necessary for oral cavity epithelium generation and plays a significant role in cell behavior. There is also a study that indicated that laminins with $\alpha 5$ chain are essential for several biological behaviors among epidermal cells (43). These studies all showed that laminin $\alpha 5$ is a crucial factor in biological functions and participates in several cell behaviors. The data of the present study suggest that laminin $\alpha 5$ is associated with epidural fibrosis and might modulate fibroblast proliferation through the PI3K/AKT/mTOR signaling pathway.

In conclusion, the present study confirmed the association between laminin $\alpha 5$ and epidural fibrosis. Furthermore, a possible mechanism was also found that laminin $\alpha 5 \mathrm{might}$ modulate fibroblast proliferation through the PI3K/AKT/mTOR signaling pathway. The results of this study could indicate a potential treatment to prevent epidural fibrosis. However, due to time limitations in this study, there are also more complex experiments have not been performed such as using an inducible laminin $\alpha 5$ knockout mouse which would take 1-2 years. In the future, the present authors may perform this experiment to aid further conclusions and find out more regarding the potential mechanism.

\section{Acknowledgements}

Not applicable.

\section{Funding}

The present study was supported by the National Natural Science Foundation of China (grant nos. 81772331, 81371971 and 81271994), the Jiangsu Provincial Medical Youth Talent (grant no. QNRC2016344), the Six talent peaks project of Jiangsu Province (grant no. 2015-WSN-108 and 2015 WSN 110), the Jiangsu Provincial 333 Project Foundation (grant no. BRA2018194), the Social Development Projects of Yangzhou Science and Technology Bureau (grant no. YZ2017073), the China Postdoctoral Science Foundation (grant no. 2016M590431) and the Jiangsu Provincial Medical Innovation Team (grant no. CXTDB2017004).

\section{Availability of data and materials}

The datasets used and/or analyzed during the current study are available from the corresponding author on reasonable request.

\section{Authors' contributions}

PL designed the research, performed the experiments and wrote the manuscript. HC contributed to the reagents, materials, analysis tools and analyzed the data. LY prepared the figures and tables. YS helped design the experiments, prepared the animal models and collected the tissue, and reviewed the drafts of the manuscript.

\section{Ethics approval and consent to participate}

The present study protocol was approved by the Research Ethics Committee of the Northern Jiangsu People's Hospital (Yangzhou, China) and written informed consent was obtained from all the participants for their tissues to be used for the purposes of this research.

\section{Patient consent for publication}

Not applicable.

\section{Competing interests}

The authors declare that they have no competing interests.

\section{References}

1. Burton CV, Kirkaldy-Willis WH, Yong-Hing K and Heithoff KB: Causes of failure of surgery on the lumbar spine. Clin Orthop Relat Res 157: 191-199, 1981.

2. Songer MN, Rauschning W, Carson EW and Pandit SM: Analysis of peridural scar formation and its prevention after lumbar laminotomy and discectomy in dogs. Spine (Phila Pa 1976) 20: 571-580, 1995.

3. Sen O, Kizilkilic O, Aydin MV, Yalcin O, Erdogan B, Cekinmez M, Caner $\mathrm{H}$ and Altinors N: The role of closed-suction drainage in preventing epidural fibrosis and its correlation with a new grading system of epidural fibrosis based on MRI. Eur Spine J 14: 409-414, 2005.

4. Mirzai H, Eminoglu $M$ and Orguc S: Are drains useful for lumbar disc surgery? A prospective, randomized clinical study. J Spinal Disord Tech 19: 171-177, 2006.

5. Cekinmez M, Erdogan B, Tufan K, Sarica FB, Ozen O and Caner H: Is topical tissue plasminogen activator application effective on prevention of post-laminectomy epidural fibrosis? An experimental study. Neurol Res 31: 322-326, 2009.

6. Sun Y, Zhao S, Li X, Yan L, Wang J, Wang D, Chen H, Dai J and He J: Local application of rapamycin reduces epidural fibrosis after laminectomy via inhibiting fibroblast proliferation and prompting apoptosis. J Orthop Surg Res 11: 58, 2016.

7. Chen H, Yan L, Wang J, Sun Y, Li X, Zhao S, Wang D, Zhu G and Liang Y: Methotrexate prevents epidural fibrosis through endoplasmic reticulum stress signalling pathway. Eur J Pharmacol 796: 131-138, 2017.

8. Dai J, Li X, Yan L, Chen H, He J, Wang S, Wang J and Sun Y: The effect of suramin on inhibiting fibroblast proliferation and preventing epidural fibrosis after laminectomy in rats. J Orthop Surg Res 11: 108, 2016.

9. Atsuta I, Yamaza T, Yoshinari M, Goto T, Kido MA, Kagiya T, Mino S, Shimono M and Tanaka T: Ultrastructural localization of laminin-5 (gamma2 chain) in the rat peri-implant oral mucosa around a titanium-dental implant by immuno-electron microscopy. Biomaterials 26: 6280-6287, 2005

10. Durbeej M: Laminins. Cell Tissue Res 339: 259-268, 2010.

11. Burgeson RE, Chiquet M, Deutzmann R, Ekblom P, Engel J, Kleinman H, Martin GR, Meneguzzi G, Paulsson M, Sanes J, et al: A new nomenclature for the laminins. Matrix Biol 14: 209-211, 1994.

12. Domogatskaya A, Rodin S and Tryggvason K: Functional diversity of laminins. Annu Rev Cell Dev Biol 28: 523-553, 2012. 
13. Petz M, Them N, Huber H, Beug H and Mikulits W: La enhances IRES-mediated translation of laminin B1 during malignant epithelial to mesenchymal transition. Nucleic Acids Res 40: 290-302, 2012.

14. Aumailley M and Rousselle P: Laminins of the dermo-epidermal junction. Matrix Biol 18: 19-28, 1999.

15. Petz M, Them NC, Huber H and Mikulits W: PDGF enhances IRES-mediated translation of Laminin B1 by cytoplasmic accumulation of La during epithelial to mesenchymal transition. Nucleic Acids Res 40: 9738-9749, 2012.

16. Sun Y, Wang TL, Toh WS and Pei M: The role of laminins in cartilaginous tissues: From development to regeneration. Eur Cell Mater 34: 40-54, 2017.

17. Savino W, Mendes-da-Cruz DA, Golbert DC, Riederer I and Cotta-de-Almeida V: Laminin-mediated interactions in thymocyte migration and development. Front Immunol 6: 579, 2015.

18. Laperle A, Hsiao C, Lampe M, Mortier J, Saha K, Palecek SP and Masters KS: $\alpha-5$ Laminin synthesized by human pluripotent stem cells promotes self-renewal. Stem Cell Rep 5: 195-206, 2015.

19. Miner JH: Laminins and their roles in mammals. Microsc Res Tech 71: 349-356, 2008

20. Gao J, DeRouen MC, Chen CH, Nguyen M, Nguyen NT, Ido H, Harada K, Sekiguchi K, Morgan BA, Miner JH, et al: Laminin-511 is an epithelial message promoting dermal papilla development and function during early hair morphogenesis Genes Dev 22: 2111-2124, 2008.

21. Sampaolo S, Napolitano F, Tirozzi A Reccia MG, Lombardi L, Farina O, Barra A, Cirillo F, Melone MAB, Gianfrancesco F, et al: Identification of the first dominant mutation of LAMA5 gene causing a complex multisystem syndrome due to dysfunction of the extracellular matrix. J Med Genet 54: 710-720, 2017.

22. Napolitano F, Di Iorio V, Di Iorio G, Melone MAB, Gianfrancesco F, Simonelli F, Esposito T, Testa F and Sampaolo S: Early posterior vitreous detachment is associated with LAMA5 dominant mutation. Ophthalmic Genet 40: 39-42, 2019.

23. Engelman JA, Luo J and Cantley LC: The evolution of phosphatidylinositol 3-kinases as regulators of growth and metabolism. Nat Rev Genet 7: 606-619, 2006.

24. Lam L, Hu X, Aktary Z, Andrews DW and Pasdar M: Tamoxifen and ICI 182,780 increase Bcl-2 levels and inhibit growth of breast carcinoma cells by modulating PI3K/AKT, ERK and IGF-1R pathways independent of ERalpha. Breast Cancer Res Treat 118: 605-621, 2009.

25. Wang S, Li X, Yan L, Nie Q, Dai J, Chen H, Wang J and Sun Y: Tamoxifen inhibits fibroblast proliferation and prevents epidural fibrosis by regulating the AKT pathway in rats. Biochem Biophys Res Commun 497: 937-942, 2018.

26. Sun Y, Wang LX, Wang L, Sun SX, Cao XJ, Wang P and Feng L: A comparison of the effectiveness of mitomycin $\mathrm{C}$ and 5-fluorouracil in the prevention of peridural adhesion after laminectomy. J Neurosurg Spine 7: 423-428, 2007.

27. Livak KJ and Schmittgen TD: Analysis of relative gene expression data using real-time quantitative PCR and the 2(-Delta Delta C(T)) method. Methods 25: 402-408, 2001

28. Dai J, Sun Y, Yan L, Wang J, Li X and He J: Upregulation of NOXA by 10 -Hydroxycamptothecin plays a key role in inducing fibroblasts apoptosis and reducing epidural fibrosis. PeerJ 5 : e2858, 2017.

29. Jiao R, Chen H, Wan Q, Zhang X, Dai J, Li X, Yan L and Sun Y: Apigenin inhibits fibroblast proliferation and reduces epidural fibrosis by regulating $W n t 3 a / \beta$-catenin signaling pathway. J Orthop Surg Res 14: 258, 2019.
30. Li X, Chen H, Wang S, Dai J, Yan L, Wang J and Sun Y: Tacrolimus induces fibroblasts apoptosis and reduces epidural fibrosis by regulating miR-429 and its target of RhoE. Biochem Biophys Res Commun 490: 1197-1204, 2017.

31. Li X, Wang S, Dai J, Yan L, Zhao S, Wang J and Sun Y: Homoharringtonine prevents surgery-induced epidural fibrosis through endoplasmic reticulum stress signaling pathway. Eur J Pharmacol 815: 437-445, 2017.

32. Sandoval MA and Hernandez-Vaquero D: Preventing peridural fibrosis with nonsteroidal anti-inflammatory drugs. Eur Spine J 17: 451-455, 2008.

33. Pozzi A, Yurchenco PD and Iozzo RV: The nature and biology of basement membranes. Matrix Biol 57-58: 1-11, 2017.

34. Hynes RO: The evolution of metazoan extracellular matrix. J Cell Biol 196: 671-679, 2012.

35. Spenle C, Simon-Assmann P, Orend G and Miner JH: Laminin $\alpha 5$ guides tissue patterning and organogenesis. Cell Adh Migr 7: 90-100, 2013.

36. Fukumoto S, Miner JH, Ida H, Fukumoto E, Yuasa K, Miyazaki H, Hoffman MP and Yamada Y: Laminin alpha5 is required for dental epithelium growth and polarity and the development of tooth bud and shape. J Biol Chem 281: 5008-5016, 2006.

37. Sharma N, Nanta R, Sharma J, Gunewardena S, Singh KP, Shankar S and Srivastava RK: PI3K/AKT/mTOR and sonic hedgehog pathways cooperate together to inhibit human pancreatic cancer stem cell characteristics and tumor growth. Oncotarget 6: 32039-32060, 2015.

38. Su JS, Woods SM and Ronen SM: Metabolic consequences of treatment with AKT inhibitor perifosine in breast cancer cells. NMR Biomed 25: 379-388, 2012.

39. Bundschuh CV, Modic MT, Ross JS, Masaryk TJ and Bohlman H: Epidural fibrosis and recurrent disk herniation in the lumbar spine: MR imaging assessment. AJR Am J Roentgenol 150: 923-932, 1988.

40. Ritie L, Spenle C, Lacroute J, Bolcato-Bellemin AL, Lefebvre O, Bole-Feysot C, Jost B, Klein A, Arnold C, Kedinger M, et al: Abnormal Wnt and PI3Kinase signaling in the malformed intestine of lama5 deficient mice. PLoS One 7: e37710, 2012

41. Xia H, Nho RS, Kahm J, Kleidon J and Henke CA: Focal adhesion kinase is upstream of phosphatidylinositol 3-kinase/Akt in regulating fibroblast survival in response to contraction of type I collagen matrices via a beta 1 integrin viability signaling pathway. J Biol Chem 279: 33024-33034, 2004

42. Santos AR, Corredor RG, Obeso BA, Trakhtenberg EF, Wang Y, Ponmattam J, Dvoriantchikova G, Ivanov D, Shestopalov VI, Goldberg JL, et al: $\beta 1$ integrin-focal adhesion kinase (FAK) signaling modulates retinal ganglion cell (RGC) survival. PLoS One 7: e48332, 2012.

43. Wegner J, Loser K, Apsite G, Nischt R, Eckes B, Krieg T, Werner $S$ and Sorokin L: Laminin $\alpha 5$ in the keratinocyte basement membrane is required for epidermal-dermal intercommunication. Matrix Biol 56: 24-41, 2016.

This work is licensed under a Creative Commons Attribution-NonCommercial-NoDerivatives 4.0 International (CC BY-NC-ND 4.0) License. 